\title{
CONCEPÇÕES SOBRE IDENTIDADE SOCIAL DA PESSOA EM SOFRIMENTO MENTAL NOS ESTUDOS DE ENFERMAGEM*
}

Yanna Cristina Moraes Lira Nascimentoํㅜ, Mércia Zeviani Brêda², Regina Maria dos Santos², Kely Regina da Silva Lima $^{3}$

\begin{abstract}
RESUMO: Revisão integrativa objetivou apresentar a síntese do conhecimento publicado por enfermeiros sobre as concepções de identidade social da pessoa em sofrimento mental. Foram incluídos 15 artigos das bases de dados selecionadas e submetidos à adaptação do questionário validado por Ursi-2005 para construção de quadro síntese. Incluíram-se publicações de 2001 a 2011 que apresentasse em sua autoria enfermeiros, utilizou-se para a busca os descritores identificação social and saúde mental and enfermagem. Os resultados apontam que $66,7 \%$ dos fenômenos estudados nos artigos referem-se a questões étnicas e todas as publicações revelam elementos que contribuem para a configuração da identidade social como sentimento de pertença, desenraizamento, adaptação a uma nova cultura, apoio social, acontecimentos políticos ou históricosociais, estigmatização e autonomia. A ausência da concepção teórica de identidade social nos estudos não impediu elencar contribuições ao aperfeiçoamento de pesquisas e às práticas de enfermagem destinadas às pessoas em sofrimento mental.

DESCRITORES: Identificação social; Saúde mental; Enfermagem; Sofrimento mental.

\section{CONCEPTIONS REGARDING SOCIAL IDENTITY OF THE PERSON WITH MENTAL SUFFERING IN NURSING STUDIES}

ABSTRACT: This integrative review aimed to present a summary of the knowledge published by nurses on the conceptions of the social identity of the person with mental suffering. It included 15 articles selected from the databases, submitted to the adaptation of the questionnaire validated by Ursi-2005, for construction of a summary table. Publications from 2001 to 2011 were included which presented nurses among their authors; for the search, the descriptors were social identification and mental health and nursing. The results indicate that $66.7 \%$ of the phenomena studied in the articles refer to issues of ethnicity and all the publications revealed elements which contribute to the configuration of the social identity as a feeling of belonging, uprootedness, adaptation to a new culture, social support, political or historical-social happenings, stigmatization and autonomy. The absence of a theoretical conception of social identity in the studies did not stop the authors from listing contributions to the improvement of research and to nursing practices directed at people with mental suffering.

DESCRIPTORS: Social identification; Mental health; Nursing; Mental suffering.

\section{CONCEPCIONES SOBRE IDENTIDAD SOCIAL DE LA PERSONA EN SUFRIMIENTO MENTAL EN LOS ESTUDIOS DE ENFERMERÍA}

RESUMEN: Revisión integrativa cuya finalidad fue presentar la síntesis del conocimiento publicado por enfermeros acerca de las concepciones de identidad social de la persona en sufrimiento mental. Fueron incluidos 15 artículos de las bases de datos seleccionadas y sometidos a la adaptación del cuestionario validado por Ursi-2005 para construcción de cuadro síntese. Se incluyeron publicaciones de 2001 a 2011 que presentasen en su autoría enfermeros y para la búsqueda fueron utilizados los descriptores identificación social and salud mental and enfermería. Los resultados apuntan que $66,7 \%$ de los fenómenos estudiados en los artículos se relacionan a cuestiones étnicas y todas las publicaciones revelan elementos que contribuyen para la configuración de la identidad social como sentimiento de pertenecer, desarraigar, adaptación a una nueva cultura, apoyo social, acontecimientos políticos o histórico-sociales, estigmatización y autonomía. La ausencia de la concepción teórica de identidad social en los estudios no ha impedido elencar contribuciones al perfeccionamiento de investigaciónes y a las prácticas de enfermería destinadas a las personas en sufrimiento mental. DESCRIPTORES: Identificación social; Salud mental; Enfermería; Sufrimiento mental.

\footnotetext{
*Originado a partir de Disciplina do Mestrado do Programa de Pós-Graduação em Enfermagem da Universidade Federal de Alagoas.

${ }^{1}$ Enfermeira. Mestre em Enfermagem. Professora da Escola de Enfermagem e Farmácia da Universidade Federal de Alagoas.

${ }^{2}$ Enfermeira. Doutora em Enfermagem. Professora da Escola de Enfermagem e Farmácia e do Programa de Pós-Graduação em Enfermagem da Universidade Federal de Alagoas.

${ }^{3}$ Enfermeira. Mestre em Enfermagem. Professora do Curso de Graduação em Enfermagem da Sociedade de Ensino Universitário do Nordeste.
}

Autor correspondente:

Yanna Cristina Moraes Lira Nascimento

Universidade Federal de Alagoas

Qd H, R. 12, 60 - 57073-020 - Maceió-AL-Brasil

E-mail: ycristina01@gmail.com
Recebido: 25/06/2012

Aprovado: 20/02/2013

Cogitare Enferm. 2013 Jan/Mar; 18(1):102-8 


\section{INTRODUÇÃO}

As concepções de identidade estão interligadas aos fenômenos sociais contemporâneos tornando-se alvo de numerosos estudos, com diversidade considerável de significados, referenciais e campos de aplicação. O termo identidade, utilizado inicialmente por filósofos, nasceU a partir da crise de pertencimento e do esforço em transpor a lacuna existente entre "o que se deve ser e o que se é"(1:618). Identidade é o que nós somos, de onde nós provimos, "é o ambiente no qual gostos, desejos, opiniões e aspirações fazem sentido"(2:54).

A "identidade é um processo socialmente construído de forma complexa, que ocorre em dado momento histórico"(3:48), com reflexos em sua concepção ao longo do tempo, que ora a compreende como estática, ora com possibilidades de mudanças limitadas e atreladas ao contexto vivido; e ora em incessante mudança ${ }^{(4)}$. Esta ideia de movimento e dinamicidade dos processos de constituição da identidade, própria da contemporaneidade, se intensifica com a globalização. Juntamente com este contexto, a necessidade de exercer diferentes papéis, em diversos contextos, acaba por favorecer o surgimento de inúmeras identidades.

Neste sentido, a identidade, é entendida como metamorfose resultante de um processo histórico que articula toda vivência, é construção e desconstrução constante, em que

cada pessoa encarna as relações sociais, configurando uma identidade pessoal. Uma história de vida. Um projeto de vida. Uma vida que nem sempre-é-vivida, no emaranhado das relações sociais $^{(5: 127)}$.

$\mathrm{Na}$ atualidade é indiscutível a busca da identidade como representação e construção do 'eu único', referência de liberdade, felicidade e cidadania. A

identidade passa a resgatar a individualidade como valor cardeal e com ela a multiplicidade e o movimento dos fenômenos para superar a razão formal da lógica do um ${ }^{(6: 19)}$.

Isso revela que as concepções de identidade estática ou em constante mutação, longe de se anularem, se complementam e permitem concebê-las como 'Identificações em curso'. Isto é, identidade, ao mesmo tempo, se transforma e afirma um modo de ser, pois, caso contrário, o risco que se corre é conceber identidade de forma extremamente mutável e volátil em que não se pode determinar nada com precisão, ou em outro extremo, uma identidade clichê sem possibilidade de mudança ${ }^{(6)}$.

Tais reflexões se tornam relevantes para a compreensão de que a identidade, quer social, psicossocial, cultural ou individual, é constantemente transformada. Mas, muitas das suas características podem estar mantidas, ou aprimoradas, com o intuito de afirmar a existência da pessoa no mundo. Assim, a forma de compreender a identidade social influencia diretamente na saúde dos sujeitos, seja na autoestima, no reconhecimento social, na formação de estigmas, na exclusão social; enfim, nos modos de viver 'saudável' em sociedade ${ }^{(7)}$.

De tal modo, estudos de identidade social no campo da saúde, e mais especificamente na enfermagem em saúde mental, têm sido acessados, estimulando a revisão de paradigmas internalizados e de práticas de saúde guiadas por tais paradigmas. Neste contexto, esta revisão integrativa objetiva apresentar a síntese do conhecimento publicado por enfermeiros sobre as concepções de identidade social da pessoa em sofrimento mental.

\section{MÉTODO}

A revisão integrativa é um método de pesquisa que permite a busca, a avaliação crítica e a síntese das evidências disponíveis do tema investigado seguindo algumas etapas; seu produto final é o estado atual do conhecimento que poderão direcionar futuras pesqui$\operatorname{sas}^{(8-9)}$. Os procedimentos adotados para esta revisão, após a definição do objetivo, foram: a descrição dos critérios de inclusão e exclusão; a definição das fontes de pesquisa (bases de dados); a execução da pesquisa; a seleção e a análise dos artigos.

Constituíram critérios de inclusão: artigos publicados entre 2001 e 2011 nos periódicos nacionais e internacionais, que tivessem a participação de enfermeiros em sua autoria, que tratassem de identidade social e que estivessem disponíveis por meio eletrônico. Foram excluídos os artigos que tratavam de identidade profissional ou organizacional.

A coleta de dados foi realizada no mês de junho e julho de 2011 nas bases de dados da Literatura Latino-Americano e do Caribe em Ciências da Saúde (LILACS), MEDLINE/Pubmed, Scientific Electronic Library Online (SciELO), IBECS e BDEnf, através do cruzamento entre descritores. Utilizou-se como estratégia de busca: identificação social and saúde mental and enfermagem. Resultaram 
22 artigos, destes 7 não estavam disponíveis, deste modo, foram incluídos neste estudo 15 artigos.

Para a coleta utilizou-se a adaptação do questionário validado por Ursi ${ }^{(8)}$. Para análise e interpretação dos dados foi construído inicialmente um quadro com a identificação do estudo quanto à Base de Dados, o periódico e ano de publicação, e o tipo de estudo ou abordagem metodológica definida pelos autores.

Em seguida nova leitura dos artigos foi realizada para selecionar os fenômenos estudados de modo a organizá-los em um quadro síntese. Assim, foram identificados elementos que contribuem na configuração da Identidade Social e explorados na discussão dos resultados.

\section{RESULTADOS}

Todas as publicações estão indexadas em mais de uma base de dados, sendo que 93,34\% ( $\mathrm{n}=14)$ delas encontram-se na base MEDLINE/Pubmed. A maioria dos artigos é proveniente de revistas internacionais de enfermagem. Quanto ao desenho metodológico, revela multiplicidade de abordagens e tipos de estudos e evidencia-se notável crescimento $(200 \%)$ de publicações sobre identidade social escritos por enfermeiros, nos últimos seis anos (Quadro 1).

Quanto aos fenômenos estudados, o quadro 2 evidencia que a maioria trata de questões étnicas relacionadas à Identidade $(\mathrm{n}=10,66,7 \%)$. Entre estas questões étnicas, estudos com imigrantes foram os mais encontrados ( $\mathrm{n}=5,33,7 \%)$. Quanto aos artigos sobre adoecimento mental na configuração da identidade $(\mathrm{n}=5,33,7 \%)$ a maioria trata da necessidade de apoio social no processo de reabilitação $(n=2,13,4 \%)$.

Dentre os artigos do quadro 2, apenas um explicita a concepção de identidade cultural à luz do referencial teórico da Modernidade Reflexiva de Anthony Guiddens. Conceitos de identidade social não foram acessados, porém os artigos trouxeram ricos elementos que contribuem na configuração da identidade social, tais como sentimento de pertença $(n=5,33,7 \%)$, desenraizamento $(\mathrm{n}=2,13,3 \%)$, adaptação à nova cultura $(n=6,40 \%)$, apoio social $(n=5,33,7 \%)$, acontecimentos políticos e/ou históricos sociais $(\mathrm{n}=4,26,7 \%)$, estigmatização $(n=5,33,7 \%)$ e autonomia $(n=3,20 \%)$.

A frequência de estudos, quanto à contribuição para a prática da enfermagem foram considerados a partir da avaliação de suas implicações, sendo 10(67\%) com implicações diretas e cinco (33\%) com implicações indiretas.
Quadro 1 - Caracterização dos artigos sobre concepções de identidade social da pessoa em sofrimento mental, 2001-2011. Maceió, 2012

\begin{tabular}{|c|c|c|}
\hline Base de Dados & $\begin{array}{l}\text { Periódico e Ano } \\
\text { de Publicação }\end{array}$ & $\begin{array}{l}\text { Tipo/ } \\
\text { abordagem }\end{array}$ \\
\hline $\begin{array}{l}\text { M E D L I N E/ } \\
\text { Pubmed }\end{array}$ & $\begin{array}{l}\text { Nursing Inquiry } \\
\text { (2011) }\end{array}$ & Conceitual \\
\hline $\begin{array}{l}\text { M E D L I N E/ } \\
\text { Pubmed }\end{array}$ & $\begin{array}{l}\text { Issues in Mental } \\
\text { Health Nursing } \\
(2009)\end{array}$ & Narrativo \\
\hline $\begin{array}{l}\text { M E D L I N E / } \\
\text { Pubmed }\end{array}$ & $\begin{array}{l}\text { Polic, Politics \& } \\
\text { Nursing Practice } \\
(2007)\end{array}$ & Conceitual \\
\hline $\begin{array}{l}\text { M EDL I N E / } \\
\text { Pubmed }\end{array}$ & $\begin{array}{l}\text { Issues in Mental } \\
\text { Health Nursing } \\
(2004)\end{array}$ & Fenomenológico \\
\hline $\begin{array}{l}\text { M E D L I N E / } \\
\text { Pubmed }\end{array}$ & $\begin{array}{l}\text { Journal of Psycho- } \\
\text { social Nursing } \\
\text { and Mental Health } \\
\text { Services (2008) }\end{array}$ & $\begin{array}{l}\text { Baseada em } \\
\text { evidência }\end{array}$ \\
\hline $\begin{array}{l}\text { M E D L I N E/ } \\
\text { Pubmed }\end{array}$ & $\begin{array}{l}\text { Nursing Standard } \\
(2007)\end{array}$ & Reflexão \\
\hline $\begin{array}{l}\text { M E D L I N E / } \\
\text { Pubmed }\end{array}$ & $\begin{array}{l}\text { Journal of Psycho- } \\
\text { social Nursing } \\
\text { and Mental Health } \\
\text { Services (2007) }\end{array}$ & Relato e revisão \\
\hline $\begin{array}{l}\text { MEDL I N E/ } \\
\text { Pubmed }\end{array}$ & $\begin{array}{l}\text { Advances in Nur- } \\
\text { sing Science: } A N S \\
(2009)\end{array}$ & Interseccional \\
\hline $\begin{array}{l}\text { M E D L I N E/ } \\
\text { Pubmed }\end{array}$ & $\begin{array}{l}\text { Issues in Mental } \\
\text { Health Nursing } \\
(2005)\end{array}$ & Reflexão \\
\hline $\begin{array}{l}\text { M E D L I N E / } \\
\text { Pubmed }\end{array}$ & $\begin{array}{l}\text { Archives of } \\
\text { Psychiatric Nursing } \\
\text { (2008) }\end{array}$ & Revisão \\
\hline $\begin{array}{l}\text { MEDL I N E/ } \\
\text { Pubmed }\end{array}$ & $\begin{array}{l}\text { Issues in Mental } \\
\text { Health Nursing } \\
(2006)\end{array}$ & Quanti-qualitativo \\
\hline $\begin{array}{l}\text { M E D L I N E/ } \\
\text { Pubmed }\end{array}$ & $\begin{array}{l}\text { Archives of } \\
\text { Psychiatric Nur- } \\
\text { sing (2005) }\end{array}$ & $\begin{array}{c}\text { Longitudinal/ } \\
\text { correlacional }\end{array}$ \\
\hline $\begin{array}{l}\text { M EDL I N E/ } \\
\text { Pubmed }\end{array}$ & $\begin{array}{l}\text { Issues in Mental } \\
\text { Health Nursing } \\
(2008)\end{array}$ & Etnográfico \\
\hline $\begin{array}{l}\text { M EDL I N E/ } \\
\text { Pubmed }\end{array}$ & $\begin{array}{l}\text { Archives of } \\
\text { Psychiatric Nursing } \\
\text { (2009) }\end{array}$ & $\begin{array}{l}\text { Descritivo- } \\
\text { correlacional }\end{array}$ \\
\hline $\begin{array}{l}\text { SciELO/ } \\
\text { LILACS }\end{array}$ & $\begin{array}{l}\text { Rev. Esc.de } \\
\text { Enfermagem da } \\
\text { USP (2009) }\end{array}$ & $\begin{array}{l}\text { Qualitativo } \\
\text { descritivo }\end{array}$ \\
\hline
\end{tabular}


Quadro 2 - Fenômenos estudados e elementos que contribuem na configuração de identidade social da pessoa em sofrimento mental, 2001-2011. Maceió, 2012

\begin{tabular}{|l|l|}
\hline \multicolumn{1}{|c|}{ Fenômenos estudados } & \multicolumn{1}{c|}{ Elementos } \\
\hline $\begin{array}{l}\text { Reflexos do Programa de Ação de Recuperação do } \\
\text { Bem-Estar e a autogestão em saúde mental }\end{array}$ & Autonomia/ Estigmatização \\
\hline Deslocamento de imigrantes no Canadá & Sentimento de pertença/Desenraizamento \\
\hline Aculturação e stress aculturativo em latinos & Adaptação à nova cultura/ Apoio social \\
\hline $\begin{array}{l}\text { Experiência dos refugiados bósnios nos Estados } \\
\text { Unidos sobre sua adaptação à nova pátria }\end{array}$ & $\begin{array}{l}\text { Adaptação à nova cultura/ Aconteci-mentos políticos } \\
\text { e/ou históricos sociais }\end{array}$ \\
\hline Estigma e efeitos da doença mental & Estigmatização/ Autonomia \\
\hline $\begin{array}{l}\text { Compreensão dos profissionais sobre necessidades das } \\
\text { pessoas que deixaram uma seita religiosa }\end{array}$ & $\begin{array}{l}\text { Sentimento de pertença/ Autonomia/ Adaptação a } \\
\text { nova cultura }\end{array}$ \\
\hline $\begin{array}{l}\text { Cuidados de saúde mental para nativos fornecidos por } \\
\text { profissional de enfermagem não nativo }\end{array}$ & $\begin{array}{l}\text { Adaptação à nova cultura/ Aconte-cimentos políticos } \\
\text { e/ou históricos sociais }\end{array}$ \\
\hline $\begin{array}{l}\text { Síndrome Sojourner e representação da identidade } \\
\text { social de mulheres afro-americanas }\end{array}$ & $\begin{array}{l}\text { Adaptação à nova cultura/ Aconte-cimentos políticos } \\
\text { e/ou históricos sociais }\end{array}$ \\
\hline Barreiras à saúde mental entre as minorias étnicas & Estigmatização \\
\hline $\begin{array}{l}\text { Implicações da cultura no tratamento da depressão } \\
\text { entre os imigrantes coreanos na América }\end{array}$ & $\begin{array}{l}\text { Adaptação à nova cultura/ Estigmatização/ Apoio } \\
\text { social }\end{array}$ \\
\hline $\begin{array}{l}\text { Quadro Integrativo de Competências da cultura } \\
\text { marginalizada como guia de saúde }\end{array}$ & $\begin{array}{l}\text { Estigmatização/Acontecimentos políticos e/ou } \\
\text { históricos sociais }\end{array}$ \\
\hline Relação entre estresse percebido e depressão & Sentimento de pertença/ Apoio social \\
\hline Modelo multidimensional de apoio social & Apoio social \\
\hline Suicídio entre índios americanos & Sentimento de pertença \\
\hline A rede social de sujeitos em tratamento CAPSad & Apoio social \\
\hline
\end{tabular}

\section{DISCUSSÃO}

A identidade revela a pessoa ao mundo, mas é o convívio no mundo que a constrói. Pode ser responsável por todo processo de inclusão como de exclusão social. A constituição identitária é infindável e embebida por elementos que contribuem diretamente em sua configuração.

A busca por pertencer tem sido postulada como uma compulsão fundamental para o bem estar e a sobrevivência humana. Estabelecer e manter relacionamento com os outros é uma necessidade humana para o seu crescimento e desenvolvimento ${ }^{(10)}$. A sensação de pertencer é uma afirmação de identidade cultural ou étnica que reflete nas questões psicológicas, sociais, físicas e espirituais dos indivíduos, famílias ou comunidades ${ }^{(11)}$. O sentimento de pertença está relacionado à percepção de $\mathrm{si}^{(12)}$.

Assim, pessoas nascidas em determinada cultura e que não sabem como funcionam outras culturas, quando mudam de contexto necessitam adaptar-se ao novo, principalmente quando existe um sentimento de pertença sólido à anterior ${ }^{(13)}$. No entanto, para adaptar-se é necessário desenraizar-se da cultura de origem.
O 'desenraizamento' acontece independente de ter sido provocado por conflito global, religioso, perseguição étnica e racial, tirania, guerra, e incerteza econômica ${ }^{(14)}$, podendo ocorrer intrinsecamente entre as próprias fronteiras, sejam elas territoriais ou de espaços subjetivos. O desenraizamento pode ser tanto uma experiência negativa como positiva. Quando negativa, podem resultar na perda da identidade, acompanhada de violações pessoais de segurança e oportunidade, e sentimentos de dor e paralisia.

Por outro lado, quando o reflexo do desenraizamento é positivo, ele favorece estratégias para criar um sentido de si e de pertença na nova cultura, como prova de força, resiliência e protagonismo. Para tanto, na medida em que ocorre o desenraizamento, há a necessidade de outro elemento, a adaptação à nova cultura, condição básica para sentir-se pertencente ao grupo. Adaptar-se a uma nova cultura significará lidar com transições, mudar identidade, encontrar um novo emprego, adaptar-se a uma nova língua, fazer novos amigos, recuperar o sentido de normalidade. 
Isso revela que a adaptação pode ocorrer de forma explícita, ou implicitamente, quando, por exemplo, pessoas são retiradas de seus lares e involuntariamente são postas em locais fechados e submetidos a regras a qual não estão acostumadas, gerando mudanças drásticas na identidade e fazendo com que os sujeitos, vítimas do processo, reproduzam com outros o que fizeram com eles.

Adaptações abruptas a uma nova cultura podem provocar delírios, alucinações, insônia, amnésia, violentas explosões emocionais e até tentativas de suicídio $^{(13)}$. O ajustamento à nova cultura tem demonstrado risco elevado para sintomas depressivos ${ }^{(15)}$. Adaptar-se a uma nova cultura é lidar com as memórias do passado estando disponível ao aprendizado, é aceitar ou ignorar a discriminação ao longo do tempo, ou a vê-la como não ofensiva, prejudicial, ou ameaçadora ${ }^{(16)}$.

Outras formas de adaptação, dizem respeito à enculturação e aculturação. Sendo a enculturação, a recuperação de sua identidade através do processo de aprendizagem da própria cultura que fora perdida, para a revitalização de práticas tradicionais, é uma espécie de manutenção da cultura esquecida para resgatar a identidade desenraizada ${ }^{(17)}$. Enquanto que a aculturação é definida como processo de mudanças e trocas sociais e psicológicas que ocorrem quando existem encontros entre indivíduos de diferentes culturas, com alterações posteriores em qualquer um dos dois grupos ${ }^{(18)}$.

Assim como o desenraizamento, o processo de aculturação pode refletir tanto como oportunidade de crescimento, quanto de sofrimento mental, sendo que, em ambas as direções, os indivíduos passam por um período de estresse.

Durante a adaptação ocorre um estresse aculturativo, simbolizando as perdas, quando os sujeitos tentam se adaptar a um novo sistema de crenças, rotinas, e papéis sociais. Destacam-se como estressores aculturativos: um novo espaço de vida, a dificuldade financeira enfrentada, a barreira linguística, a falta de acesso aos cuidados de saúde, a estadia em bairros inseguros, as condições precárias de habitação, a falta de escolaridade, o desemprego e subemprego, a exploração e condições de trabalho perigosas, mudanças nos vínculos, as normas culturais, a discriminação ou estigma, a influencia de forças políticas e históricas e a falta de apoio social ${ }^{(18)}$.

A falta deste apoio configura-se, muitas vezes, como fator impeditivo no processo de recuperação, já que o modelo multidimensional de apoio tem a função de promover suporte informacional, favorecer apoio tangível, reforçar a estima e ofertar apoio emocional proporcionando conforto e segurança durante o período de estresse ${ }^{(19)}$. Outro elemento que interfere diretamente na adaptação de uma nova cultura, e na configuração da identidade social do sujeito são os acontecimentos políticos e/ou histórico sociais.

A memória cultural está associada com os conceitos de identidade e diferença, ou seja, uma vez membro de um grupo, este pensaria em si como sendo do mesmo sangue, e como tendo uma identidade comum, porém isto acaba representando uma ameaça com danos incalculáveis quando membros de um grupo, ou de uma classe passam a se envolver com membros de outro grupo ou de outra classe social ${ }^{(10)}$.

A memória cultural pode induzir também ao `trauma intergeracional', aquele causado por uma exposição traumática de um evento ocorrido na geração anterior que continua afetando as gerações seguintes, comum em filhos de sobreviventes de guerra, e nativos colonizadores, em que eram retraumatizados ao ouvir as atrocidades que aconteceram com seus ancestrais ${ }^{(17)}$. É preciso resgatar que vestígios de atos sociais e individuais resultantes da opressão, subordinação, dissolução e exploração exercem impacto profundo e duradouro sobre os papéis sociais, suas relações e a construção identitária de um grupo $^{(16)}$.

Outro elemento que também contribui na construção da identidade social é a presença do estigma, definido por um conjunto de crenças, pensamentos, atitudes e comportamentos negativos, que influenciam o indivíduo, ou o público em geral, ao medo, a rejeição, e a discriminação ${ }^{(20)}$. O estigma é uma barreira para aqueles que precisam de serviços de saúde mental, mas que relutam ou se recusam a procurar ajuda por causa da discriminação e do sentir-se rejeitados por outros. Muitas pessoas quando estão passando pelo adoecimento mental internalizam o estigma agindo como se o fenômeno fosse real e legítimo, acreditando que são incapazes de contribuir com a sociedade ${ }^{(21)}$.

Mais um elemento que contribui na configuração da identidade social é a autonomia. A cultura de preservação da autonomia apesar de estimular a autoconfiança, como ponto positivo na identidade do sujeito, também acaba responsabilizando-o e culpabilizando-o todas as vezes que um planejamento não é alcançado ${ }^{(13,22)}$.

Enfim, todos estes elementos abordam conteúdos que contribuem direta ou indiretamente na configuração, mesmo que instável, da identidade social. E a compreensão destes elementos trazem implicações para a prática da enfermagem, voltada à pessoa em sofrimento mental. Uma delas trata da necessidade do 
enfermeiro evitar julgamentos sobre as pessoas que recebem seus cuidados, considerando e respeitando os contextos de cuidado e os valores culturais de cada uma em particular. Tornar-se "culturalmente sensível" exige disponibilidade, respeito e flexibilidade em aceitar modos diferentes de viver.

Outra implicação diz respeito ao importante papel do profissional de enfermagem em favorecer a adaptação das pessoas frente às mudanças, bem como promover articulações junto a rede social de apoio, tal como a família.

Quanto à publicação de estudos sobre identidade, é preciso que forneçam informações claras e acessíveis aos que lidam com pessoas em sofrimento mental, cuidando para não endossar o estigma e a crença de que possuem péssimos prognósticos e pouca chance de recuperação ${ }^{(21-22)}$.

\section{CONCLUSÃO}

Nos estudos analisados não foram encontradas as concepções de identidade social, porém foram identificados elementos que, em seu conjunto, contribuem na configuração da identidade social das pessoas em sofrimento mental. Percebeu-se ainda, que a maioria dos artigos analisados trata de pesquisas etnográficas e culturais, com povos imigrantes, nativos ou marginalizados que estão suscetíveis ao adoecimento mental e, não necessariamente, pessoas que estão em acompanhamento em serviços de saúde mental.

Vale ressaltar que a escassez de trabalhos realizados por enfermeiros que tratem da identidade social das pessoas com transtorno mental acompanhadas em serviços de saúde mental aponta uma grande lacuna na investigação científica.

\section{REFERÊNCIAS}

1. Lima AF. Para a reconstrução dos conceitos de massa e identidade. Univ Psychol. 2007;6(3):613-22.

2. Taylor C. A política de reconhecimento. In: Gutman A. Multiculturalismo: examinando a política do reconhecimento. Lisboa: Instituto Piaget; 1998. p. 45-94.

3. Magalhães RCBP, Cardoso APLB. A pessoa com deficiência e a crise das identidades na contemporaneidade. Cad. Pesqui. 2010;40(139):45-61.

4. Hall S. A identidade cultural na pós-modernidade. Rio de Janeiro: DP\&A; 2005.
5. Ciampa AC. A estória do Severino e a história da Severina. São Paulo: Brasiliense; 1987.

6. Sawaia BB, organizador. As artimanhas da exclusão: análise psicossocial e ética da desigualdade social. $5^{\text {a }}$ ed. Petrópolis: Vozes; 2004.

7. Demo P. Introdução à Sociologia: complexidade, interdisciplinaridade e desigualdade social. São Paulo: Atlas; 2002.

8. Souza MT, Silva MD, Carvalho R. Revisão integrativa: o que é e como fazer. einstein. 2010;8(1):102-6.

9. Glanzmann LNF, Toledo VP. Interconsulta de enfermagem psiquiátrica: análise da produção científica. Cogitare enferm. 2012;17(1):138-43.

10. Keyes EF, Kane CF. Belonging and adapting: mental health of Bosnian refugees living in the United States. Issues Ment Health Nurs. 2004;25(8):809-31.

11. Hill DL. Relationship between sense of belonging as connectedness and suicide in American Indians. Arch Psychiatr Nurs. 2009;23(1):65-74.

12. Choenarom C, Williams RA, Hagerty BM. The role of sense of belonging and social support on stress and depression in individuals with depression. Arch Psychiatr Nurs. 2005;19(1):18-29.

13. Wallis L. Switching off the cult. Nurs Stand. 2007; 21(49):20-2.

14. Berman H, Mulcahy GA, Forchuk C, Edmunds KA, Haldenby A, Lopez R. Uprooted and displaced: a critical narrative study of homeless, Aboriginal, and newcomer girls in Canada. Issues Ment Health Nurs. 2009;30(7):418-30.

15. Park SY, Bernstein KS. Depression and Korean American immigrants. Arch Psychiatr Nurs. 2008;22(1):12-9.

16. Lekan D. Sojourner syndrome and health disparities in African American women. Adv. Nurs. Sci. 2009;32(4):307-21.

17. Barnard AG. Providing psychiatric-mental health care for Native Americans: lessons learned by a non-Native American PMHNP. J Psychosoc Nurs Ment Health Serv. 2007;45(5):30-5.

18. Caplan S. Latinos, acculturation, and acculturative stress: a dimensional concept analysis. Policy Polit Nurs Pract. 2007;8(2):93-106. 
19. Carless D, Douglas K. Social support for and through exercise and sport in a sample of men with serious mental illness. Issues Ment Health Nurs. 2008;29(11):1179-99.

20. Gary FA. Stigma: barrier to mental health care among ethnic minorities. Issues Ment Health Nurs. 2005;26(10):979-99.

21. Corrigan PW, Wassel A. Understanding and influencing the stigma of mental illness. J Psychosoc Nurs Ment Health Serv. 2008;46(1):42-8.

22. Scott A, Wilson L. Valued identities and deficit identities: wellness recovery action planning and selfmanagement in mental health. Nurs Inq. 2011;18(1):40-9. 